\title{
VEHICLE-INFRASTRUCTURE COOPERATIVE SYSTEMS FOR INTERSECTION COLLISION AVOIDANCE: DRIVER ASSESSMENT CHALLENGES
}

\author{
V. Inman \\ A.J. Nedzesky \\ T. Shafer \\ SAIC Transportation Research Division
}

\begin{abstract}
Summary: According to National Highway Traffic Safety Administration (NHTSA, 1998) data, there were 37,280 crashes that involved fatalities in 1997. Of these crashes, 8,571 were related to intersections. The fatal crashes at intersection were about evenly divided among noncontrolled intersections, signal controlled intersections, and stop sign controlled intersection.
\end{abstract}

In addition to fatal crashes, almost 1 million injury crashes occur at intersections annually, and there are about 1.7 million police reported crashes at intersection each year.

Various programs have proposed alternative countermeasures to reduce the number of crashes and fatalities at intersections. Conventional countermeasures such as protected left turn signals are effective and fairly well understood. However, these countermeasures alone will not eliminate intersection crashes because they do not address factors such as willful and unintentional red-light and stop sign violations, gap acceptance problems associated with older drivers, and sight distance problems at intersections that may not warrant traffic signals.

The Federal Highway Administration is pursuing infrastructure based ITS solutions to address crashes at intersections. Initially these solutions will not require changes to vehicles. It is anticipated that in the future, some of these solutions could be integrated into in-vehicle ITS systems to enable either in-vehicle warnings or automated crash avoidance systems.

Four types of intersection-infrastructure systems are envision: (1) traffic signal violation warning, (2) stop sign violation warning, (3) traffic signal left turn assistances, and (4) stop sign movement assistance. Each of these systems is described briefly, and a preliminary list of the driver behavior issues associated with each is identified.

The challenge for the design of these systems is similar to that for other areas of highway and vehicle design - how to assess driver performance and behavior with these systems before the systems are fielded. Various assessment techniques are discussed in association with the advantages and disadvantages of each. The FHWA human-centered research approach for intersection-infrastructure solutions is presented. 